\title{
IMPLEMENTAÇÃO BIM AO NÍVEL DO LICENCIAMENTO MUNICIPAL: VERIFICAÇÃO AUTOMATIZADA DO RGEU
}

\author{
Natália Gualberto $^{(1)}$, João Pedro Couto ${ }^{(1)}$, Miguel Azenha ${ }^{(1)}$ \\ (1) Universidade do Minho, Guimarães
}

\begin{abstract}
Resumo
O setor da construção civil tem registado um crescente desenvolvimento no que se refere à metodologia 'Building Information Modelling' (BIM) e aos seus diversos usos e aplicações. A verificação de regras, no âmbito do Licenciamento de Obras, é uma das vertentes que se encontra em franco desenvolvimento a nível mundial. Neste contexto, o presente artigo que baseia numa dissertação de mestrado desenvolvida na Universidade do Minho, elaborada em colaboração com a Gaiurb EM, aborda a utilização da verificação automatizada de regras com o intuito de entender como esta pode ser implementada pelas Câmaras Municipais. Foi desenvolvido um caso de estudo posteriormente testado para a verificação do cumprimento das regras estabelecidas no Decreto-Lei n. ${ }^{\circ}$ 220/2008, de 12 de novembro, que define o Regulamento Geral das Edificações Urbanas (RGEU). Para isso, foi necessário classificar as regras previstas no RGEU quanto à possibilidade de serem interpretadas e verificadas através de um software, e posteriormente testar a verificação automatizada através do modelo do caso de estudo, possibilitando compreender os desafios gerados pela verificação automatizada, como a interpretação das regras escolhidas, a sua inserção num software de verificação e as necessidades de informação que o modelo precisa conter para que a verificação ocorra corretamente. Desta forma, foi possível sugerir propostas para a implementação do uso da verificação automatizada de regras para licenciamento, no caso particular do RGEU.
\end{abstract}

\section{Introdução}

A crescente adoção da metodologia 'Building Information Modelling' (BIM) nos processos de projeto, construção e gestão do edificado a nível nacional, está a despertar cada vez mais interesse em vários contextos nos quais até o momento não tem havido desenvolvimentos relevantes. Um desses contextos é precisamente o das Autarquias, mais concretamente, nas Câmaras Municipais. É possível identificar vários usos BIM no contexto Autárquico, mas este artigo foca-se no licenciamento de obras e a sua possível melhoria com o uso do BIM.

A verificação automatizada de parâmetros normativos por meio de regras introduzidas nos sistemas computacionais tem-se mostrado eficiente, na medida em que reduz o tempo de 
verificação e análise dos projetos e ainda pode contribuir para aumentar a precisão dos resultados [1]. Deste modo, no que diz respeito aos processos de licenciamento, a junção da utilização de modelos BIM e sua devida verificação por softwares específicos, que utilizam um conjunto de regras definidas baseadas em leis e normas locais em vigor, pode contribuir não só para uma maior conformidade dos projetos, como também para uma maior rapidez na sua análise, gerando maior produtividade e menos tempo na atribuição de alvarás de construção. Este ponto vai ao encontro de um importante objetivo do Decreto-Lei $\mathrm{n}^{\circ}$ 136/2014, de 9 de setembro, referente ao Regime Jurídico da Urbanização e Edificação (RJUE), que visa a simplificação dos processos urbanísticos, na busca por um processo mais rápido, claro e objetivo, mas sem perda de qualidade e integridade das construções e do controle necessário que os agentes públicos detêm [2].

No que se refere aos trabalhos de investigação ligados ao uso do BIM para Licenciamento de Obras pelo mundo, há alguns trabalhos que podem ser citados, como é o caso da tese de doutoramento realizada em 2009 por João Poças Martins, que se debruçou sobre aspetos ligados ao contexto de licenciamento, com exemplos na verificação de regras de projetos de sistemas de distribuição predial de água [3]. Refere-se também outro estudo para verificação normativa no contexto de planos de acessibilidades [1]. É interessante salientar que neste estudo foram utilizadas quatro bases normativas, o Decreto-Lei 163/2006 de 8 de Agosto de Portugal, a norma ISO/FDIS-21542, a '2010 ADA standards for Accessible Design', dos EUA e o 'Approved Document $M$ - Access to and Use of Buildings', do Reino Unido. Estas foram classificadas quanto a possibilidade de inserção das suas regras em software de verificação automatizada. No mundo, Singapura destaca-se pela utilização do BIM em contexto de licenciamento de obras desde 2008, vindo a aprimorar não apenas sua plataforma de verificação automática de projetos, como também as diretrizes que os modelos devem seguir para uma correta verificação [4]. Além de Singapura, nos EUA já existem iniciativas na busca pela verificação automatica de modelos BIM, como por exemplo os projetos SMARTcodes e AutoCodes [5,6]. No Brasil, há um exemplo em desenvolvimento na cidade de Salvador, Bahia, com uma plataforma para verificação de regras e liberação de licenciamentos através da núvem, para modelos BIM [7].

No contexto do presente artigo, estudou-se Regulamento Geral das Edificações Urbanas (RGEU), descrito pelo Decreto-Lei $\mathrm{n}^{\mathrm{o}} 38$ 382, de 7 de agosto de 1951, e todas as suas atualizações, até à sua atual redação descrita no Decreto-Lei n. ${ }^{\circ} 220 / 2008$, de 12 de novembro [8]. A escolha deste documento como base das regras para a verificação automatizada através de um software de um modelo em BIM, deu-se pelo facto de o RGEU ser um documento de abrangência nacional, comum a todas as Câmaras Municipais e a todos os projetos construídos em Portugal. Apresenta-se a metodologia de implementação em software de verificação de regras em modelos BIM, discutindo-se aspetos relacionados com a tradução das regras do RGEU para algoritmos automatizáveis, bem como as regras de modelação recomendáveis para o efeito. Finalmente, apresenta-se um caso de estudo em que foi aplicada a metodologia desenvolvida. 


\section{Preparação e procedimentos para a verificação automatizada de regras}

\subsection{Metodologia}

O trabalho aqui reportado foi aplicado a um caso de estudo, genericamente descrito na secção 3, e modelado com o software Revit®, da Autodesk®. A implementação e verificação de regras foi efetuada com o Solibri Model Cheker® (SMC), onde foram inseridas algumas regras/normas do RGEU. O SMC é um software que tem como funcionalidades analisar modelos BIM tendo por base um conjunto de regras. Estas regras visam identificar e avisar sobre potenciais problemas, conflitos ou violações que possam surgir num determinado modelo de informação [9]. Este software foi desenvolvido pela Solibri Inc® e foi escolhido para este estudo pela sua facilidade de utilização e funcionalidades específicas de verificação (incluindo um editor de regras a verificar).

\subsection{Análise das disposições do RGEU}

Iniciou-se o processo de implementação com a leitura crítica e classificação das prescrições do RGEU, visando identificar o conjunto de regras escritas que são passíveis de implementação direta para verificação automática; o conjunto de regras a serem reformuladas, de forma a permitir a sua implementação/verificação; e o conjunto de regras que não são passíveis de implementação no software adotado, em virtude da subjetividade inerente à sua aplicação. Foi então adotada uma classificação de regras conforme descrito de seguida:

- $\quad$ NA - Quando a regra estabelece algo que não pode ser analisado pelo modelo;

- SIM - Para regras que são verificáveis de maneira automatizada;

- $\quad$ SIM_* - Para regras que são parcialmente verificáveis de maneira automatizada;

- $\quad \mathrm{NÃO}$ - Para regras que não são verificáveis de maneira automatizada.

De seguida apresentam-se e comenta-se alguns exemplos de regras do RGEU e as suas respetivas classificações:

Quadro 1: Regra classificada como NA

\begin{tabular}{|} 
Artigo 80: \\
$\begin{array}{c}\text { A utilização de qualquer edificação nova, reconstruída, ampliada ou } \\
\text { alterada, quando da alteração resultem modificações importantes nas } \\
\text { suas características, carece de licença municipal. }\end{array}$ \\
\hline Classificação: & NA (Não Aplica) \\
\hline
\end{tabular}

A regra acima destacada trata de algo que não pode ser analisado pelo modelo do projeto. Esta classificação é comum nas disposições de natureza administrativa, e também nos capítulos do Regulamento que falam sobre o decurso das obras e sanções diversas. 
Quadro 2: Regra classificada como SIM

\begin{tabular}{|c|c|}
\hline \multicolumn{2}{|l|}{ Artigo 28ㅇ: } \\
\hline \multicolumn{2}{|c|}{$\begin{array}{l}\text { Nas edificações construídas com estruturas independentes de betão armado ou metálicas, as } \\
\text { espessuras das paredes de simples preenchimento das malhas verticais das estruturas, quando } \\
\text { de alvenaria de pedra ou tijolo, poderão ser reduzidas até aos valores mínimos de cada grupo } \\
\text { fixados no artigo } 25 \text { o, desde que o menor vão livre da parede entre os elementos horizontais ou } \\
\text { verticais da estrutura não exceda 3,50m. }\end{array}$} \\
\hline Classificação: & SIM \\
\hline
\end{tabular}

Esta classificação dá-se quando a regra é objetiva, escrita de maneira clara e fácil tradução para um software de verificação automática.

Quadro 3: Regra classificada como SIM*

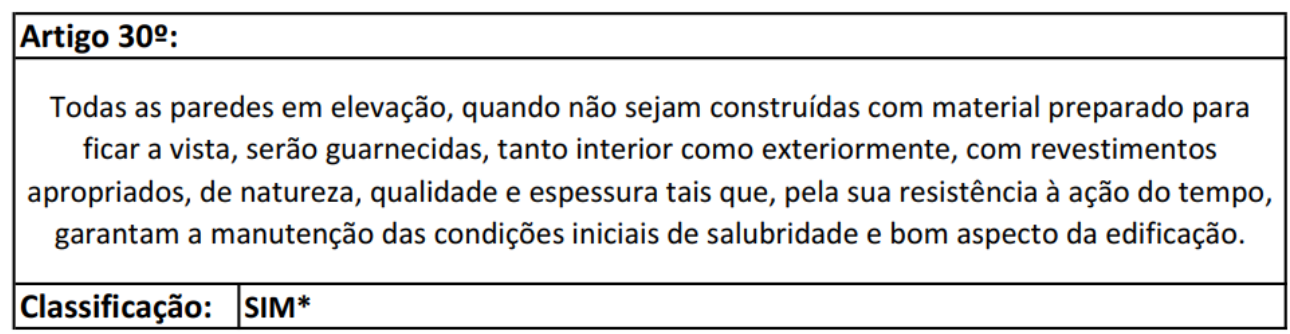

Esta classificação dá-se quando parte da regra pode ser avaliada por um software. É possível que com uma revisão textual do documento base de regras (RGEU), algumas destas possam ser reescritas, de forma que seja possível uma verificação automatizada total.

Quadro 4: Regra classificada como NÃO

\begin{tabular}{|l|}
\hline Artigo 1280: \\
\hline $\begin{array}{c}\text { As edificações serão delineadas e construídas de forma a ficar sempre } \\
\text { assegurada a sua solidez, e serão permanentemente mantidas em estado } \\
\text { de não poderem constiruir perigo para a segurança pública e dos seus } \\
\text { ocupantes ou para a dos prédios vizinhos. }\end{array}$ \\
\hline Classificação:
\end{tabular}

Esta classificação é atribuída quando a regra não pode ser verificada de forma automatizada. Da análise efetuada, concluiu-se que, das 254 regras que compõem o RGEU, 97 foram classificadas como "NA", 101 classificadas como "SIM", 39 como "SIM_*" e 17 como "NÃO". Ou seja, 55\% das regras foram classificadas como verificáveis de forma automatiza, total ou parcialmente.

Ainda sobre a classificação das regras quanto à sua capacidade de automatização, considera-se necessário também uma segunda classificação, agora relativa à possibilidade de estas serem implementadas através do SMC. O software permite a edição dos parâmetros que compõem as suas regras, como referido no ponto 2.1 , porém não permite a edição da programação da regra através de código (limitando as edições a metodologias de 'alto-nível'). Isto acaba por fazer com que algumas regras que seriam verificáveis de forma automatizada não possam ser 
verificadas através do SMC. Foi então efetuada uma classificação das regras nesse contexto, com base na classificação definida por [10]:

- D - Implementação direta no SMC - quando já existe uma regra dentro do SMC que trata do mesmo ponto, precisando apenas da alteração de alguns parâmetros;

- $\quad \mathrm{P}$ - Implementação parcial no SMC - quando já existe uma regra dentro do SMC que trata de uma parte do mesmo ponto;

- V - Visual - quando a verificação não é possível pelo SMC, porém o software pode emitir um alerta e posicionar a visualização do modelo de forma que o agente camarário que analisa o projeto possa avaliar de maneira visual;

- $\quad \mathrm{N}$ - Não há como analisar a regra pelo SMC.

É importante referir que esta classificação não substitui a anterior, sendo cumulativa com a mesma, e melhorando o entendimento quanto à automatização das regras, dentro do software escolhido para sua verificação. Apresenta-se na Figura 1 uma reprodução parcial da análise efetuada, a título exemplificativo, sendo que para a consulta da análise completa recomenda-se a leitura da dissertação de mestrado que esteve na génese do presente artigo [11].

\begin{tabular}{|c|c|c|c|c|c|c|}
\hline 옹 & 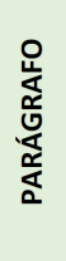 & AUTOMATIZÁVEL & $\begin{array}{l}\text { 엄 } \\
\text { 点 } \\
\text { 岃 }\end{array}$ & 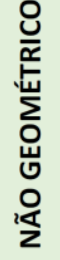 & $\begin{array}{c}\text { CLASSIF. } \\
\text { SMC }\end{array}$ & OBSERVAÇÃO \\
\hline 6 & 6 & SIM & & $x$ & D & $\begin{array}{c}\text { Usar o "Space Usage" para } \\
\text { verificação }\end{array}$ \\
\hline 23 & 23 & SIM_* & $x$ & & $P$ & \\
\hline 24 & 24 & SIM_* & $x$ & & $\mathrm{P}$ & \\
\hline 26 & 1) & SIM_* & $x$ & $x$ & $P$ & \\
\hline 30 & 30 & $\mathrm{SIM}_{-}^{*}$ & & $x$ & $\mathrm{~V}$ & \\
\hline 30 & $\S 1$ & SIM_* & & $x$ & $P$ & \\
\hline
\end{tabular}

Figura 1: Lista parcial de regras e respetiva classificação.

\subsection{Inserção de regras no software de verificação}

Utilizando-se a função 'Ruleset Manager' do SMC, é possível aceder às regras contidas no programa e editá-las através dos seus parâmetros preexistentes. Outro elemento passível de ser editado é o grau de severidade da regra, por meio da atribuição de maior ou menor ênfase a cada uma delas, consoante a realidade singular de cada análise/projeto. Após a criação do novo ficheiro que contenha as regras modificadas, o utilizador poderá guardá-las para reutilização. Como exemplo de inserção de regras, a Figura 2 ilustra como foi editada a regra 'A65 - Altura do Ambiente', no espaço 'Workspace' do 'Ruleset Manager'. 


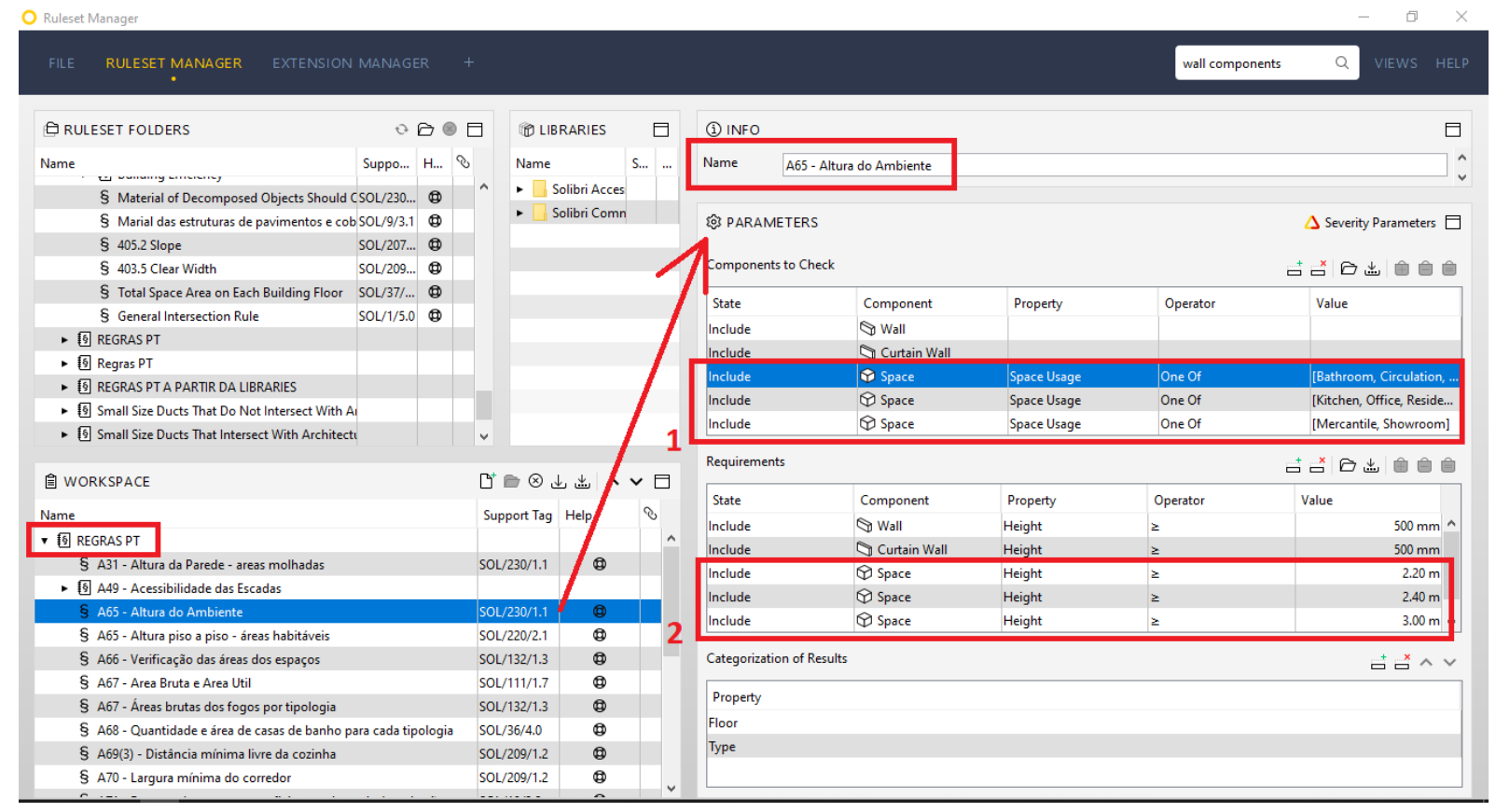

Figura 2: Edição da regra "A65 - Altura do Ambiente".

Após escolha da regra base do próprio SMC (Support Tagg SOL/230/1.1) que se adequava à regra escrita do RGEU, foram inseridos 4 novos parâmetros. No ponto 1 da imagem, vê-se a inclusão dos espaços a serem analisados "casa de banho; arrumos; circulação" e "cozinha; quartos" que no ponto 2 da imagem são novamente referidos, porém com a indicação de que a sua altura de pé-direito (do piso ao teto) deverá ser maior ou igual que 2,20 m para os espaços "casa de banho; arrumos; circulação" e maior ou igual que 2,40 m para os espaços "cozinha; quartos", como especificado no Artigo $65^{\circ}$ do regulamento base.

Uma vez efetuada a verificação das regras num determinado modelo, o SMC gera um relatório de avisos de quais foram os problemas detetados, com o respetivo grau de severidade e com a possibilidade de visualização no próprio modelo do problema detetado através de uma janela de visualização do modelo [1]. É possível ainda ativar ou desativar uma regra ou um conjunto de regras dentro do SMC, conforme necessidade de verificação das mesmas para o projeto. Isto ajuda na criação dos relatórios de avisos gerado pelo programa, que podem ser globais, ou divididos em temas e especialidades.

\subsection{Regras de modelação}

Para que a verificação automatiza de regras do RGEU funcione corretamente, é preciso que o modelo a ser verificado contenha características e informações paramétricas necessárias para a devida interpretação das regras pelo SMC. Com o intuito de que não faltem estas informações, foi desenvolvida a lista de verificação que se segue:

- Nome dos ambientes;

- Delimitar de área dos ambientes;

- Criar espaços por pavimento (ex.: "piso térreo", "pavimento 1", “pavimento 2"), contendo toda a área bruta do piso; 
- Classificar paredes do modelo para "externas" e "internas" (várias formas, uma delas: Uniformat II);

- Especificar os materiais de revestimento de piso e parede para casas de banho, sanitários, copas, cozinhas e locais de lavagem;

- Especificar os materiais de revestimento externo e interno de paredes;

- Classificar os elementos que compõem as casas de banho, como sanitas e lavatórios.

Além desta checklist, recomenda-se que o projetista, antes de subordinar o modelo final à verificação ao automatizada pela Câmara Municipal, utilize as próprias rulesets padrões do Solibri ${ }^{\circledR}$ para verificar se o modelo final está em conformidade quanto a não existência de intersecções de elementos, ou mesmo quanto a correta sobreposição dos modelos das diferentes disciplinas.

\section{Caso de estudo}

O imóvel utilizado como caso de estudo deste artigo pertence à Gaiurb, EM. O edifício é uma unidade inicialmente destinada a habitação unifamiliar, construída na Rua de S. Lourenço, Freguesia da Santa Marinha, na zona do "Castelo de Gaia", uma parte classificada como histórica pelo município. Apesar da residência "Casa do Castelo" consistir numa habitação histórica em reabilitação, e por isto estar subordinada ao RERU (Regime Excecional para a Reabilitação Urbana) [12], aqui foram aplicadas as verificações de regras consoante o RGEU. O modelo BIM produzido está ilustrado na Figura 3.

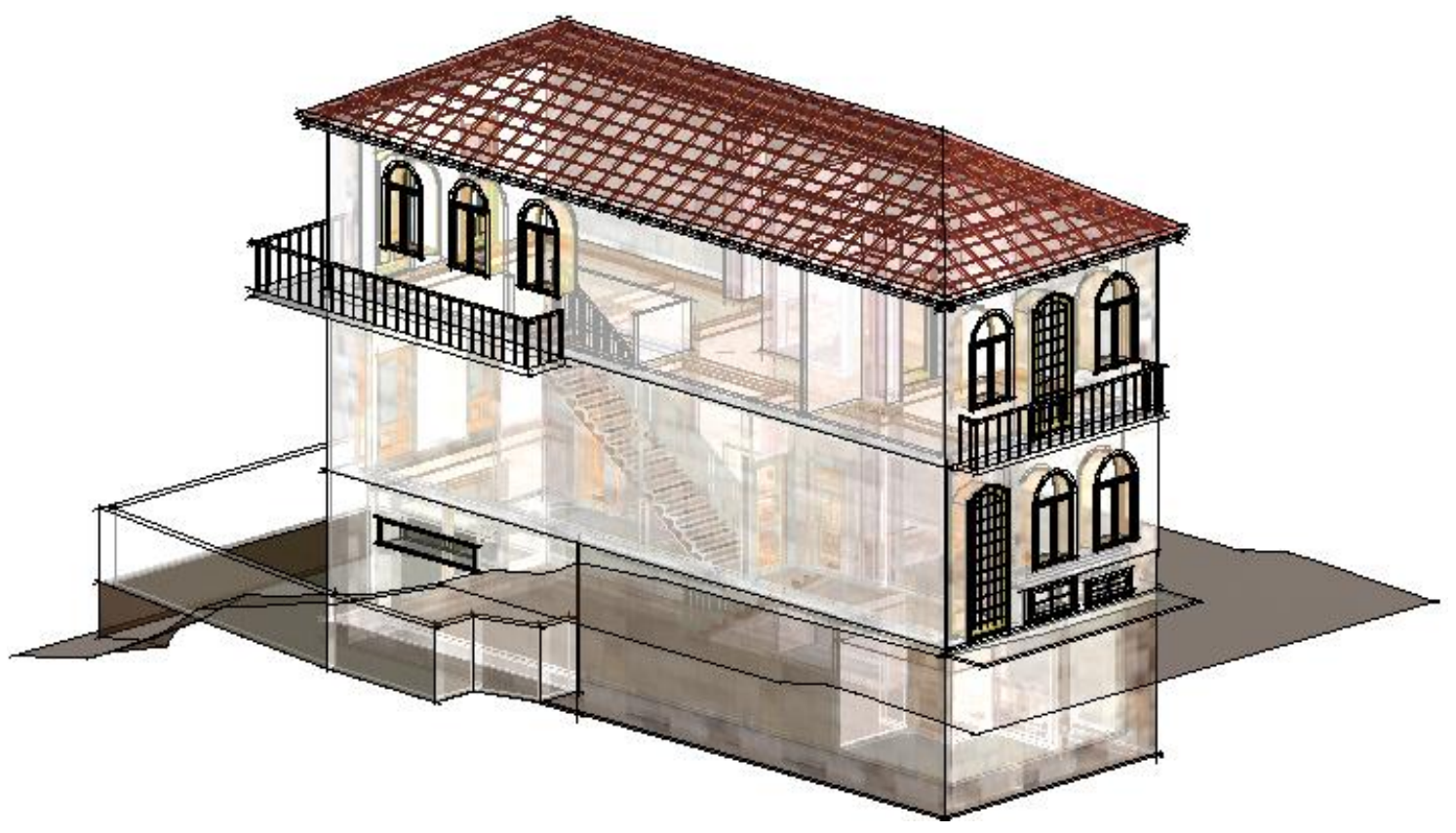

Figura 3: Modelo Casa do Castelo (frente).

Após todo o processo de preparação para a análise da verificação de regras automatizada [11], o ficheiro do modelo criado no Revit ${ }^{\circledR}$ foi exportado em formato IFC para o SMC. Dentro do 
software, diante das 55 regras apontadas como passíveis de averiguação pelo SMC, verificouse que 15 destas poderiam ser analisadas no modelo da Casa do Castelo, seja de forma direta, parcial ou visual. As 40 regras restantes não poderiam ser aplicadas pois a diretriz pode tratar de uma tipologia diferente de habitação, ou pela inexistência do que a regra se refere no projeto, como no caso do "alojamento de animais" por exemplo, ou porque o modelo de arquitetura não contém tal informação, como por exemplo as regras que fazem referência aos projetos de hidráulica.

Foi criado um 'template' no SMC com o nome 'Regras PT', como pode ser visto na Figura 4. Dentro deste 'template' está um conjunto de regras baseadas no RGEU utilizadas para a análise do modelo da residência "Casa do Castelo" [11].

\begin{tabular}{|c|c|}
\hline Name & Support Tag \\
\hline \multicolumn{2}{|l|}{ 、专 REGRAS PT } \\
\hline$\S$ A31 - Altura da Parede - areas molhadas & SOL/230/1.1 \\
\hline \multicolumn{2}{|l|}{ V 贯 A49 - Acessibilidade das Escadas } \\
\hline$\S$ Escadas em moradias UNIFAMILIARES - Degraus e Espelhos & $\mathrm{SOL} / 210 / 3.1$ \\
\hline$\S$ Escadas em moradias COLETIVAS até 2 pisos - Degraus e Espelhos & $\mathrm{SOL} / 210 / 3.1$ \\
\hline$\S$ Escadas em moradias COLETIVAS até 2 pisos LAÇOS ENTRE PAREDES - Dẹ & $\mathrm{SSOL} / 210 / 3.1$ \\
\hline$\S$ Escadas em moradias COLETIVAS mais de 2 pisos - Degraus e Espelhos & $\mathrm{SOL} / 210 / 3.1$ \\
\hline$\S$ Escadas em moradias COLETIVAS mais de 2 pisos LANÇOS ENTRE PAREDE: & SOL/210/3.1 \\
\hline$\S$ A65 - Altura do Ambiente & $\mathrm{SOL} / 230 / 1.1$ \\
\hline$\S$ A65 - Altura piso a piso - áreas habitáveis & $\mathrm{SOL} / 220 / 2.1$ \\
\hline$\S$ A66 - Verificação das áreas dos espaços & SOL/132/1.3 \\
\hline$\S$ A67 - Area Bruta e Area Util & SOL/111/1.7 \\
\hline$\S$ A67 - Áreas brutas dos fogos por tipologia & SOL/132/1.3 \\
\hline$\S$ A68 - Quantidade e área de casas de banho para cada tipologia (T0, T1 e T2) & $\mathrm{SOL} / 36 / 4.0$ \\
\hline$\S$ A68 - Quantidade e área de casas de banho para cada tipologia (T3 e T4) & $\mathrm{SOL} / 36 / 4.0$ \\
\hline § A68 - Quantidade e área de casas de banho para cada tipologia (T5 ou +) & $\mathrm{SOL} / 36 / 4.0$ \\
\hline § A69(3) - Distância mínima livre da cozinha & SOL/209/1.2 \\
\hline$\S$ A70 - Largura mínima do corredor & SOL/209/1.2 \\
\hline § A71 - Espaços devem conter suficiente valores de área de vãos externos & SOL/19/3.3 \\
\hline$\S$ A84 - Componetes para instalações sanitárias & SOL/225/1.2 \\
\hline § A85 - Existência de instalações sanitárias & SOL/230/1.1 \\
\hline$\S$ A86 - Comunicação das inst. sanit. com a habitação & SOL/161/3.1 \\
\hline
\end{tabular}

Figura 4: 'Template' criado com designação 'Regras PT'.

Executada a verificação dos modelos através das regras criadas no SMC, foi possível gerar um relatório automático, com maior ou menor conteúdo em detalhes, em formatos PDF ou Excel, contendo as informações dos erros apontados por níveis de severidade, e a classificação de "aceite" ou "rejeitado" definida pelo agente de projeto da Câmara Municipal [11]. Para o caso de verificação automatizada de regras para licenciamento de obras, este tipo de relatório pode ser anexado à decisão final como comprovativo da verificação e justificação da atribuição ou não do licenciamento para a construção. Este documento pode ainda ser entregue ao requerente do licenciamento com o intuito do projeto ser revisto em pontos específicos, e assim possa ser aprovado quando de uma segunda análise e verificação. 


\section{Conclusão}

Com a realização deste trabalho foi possível perceber que apesar do licenciamento de obras não ser um dos Usos BIM mais difundidos atualmente, é, contudo, uma ferramenta em crescente desenvolvimento a nível mundial, e de grande interesse, pois não só resulta numa verificação mais eficiente das conformidades de projeto como também aumenta a produtividade na atividade de licenciamento, através de uma maior rapidez nas análises e verificações. O trabalho permitiu analisar a aplicabilidade de regras do RGEU num software de verificação de modelos, e implementar a respetiva análise num projeto piloto, com ênfase na necessidade da definição de práticas de modelação adequadas para submissão a verificação. As capacidades de verificação constatadas revelaram-se suficientes para justificar um investimento a este nível, permitindo uma melhor garantia da satisfação dos requisitos regulamentares, quer do ponto de vista dos projetistas, quer do ponto de vista das entidades licenciadoras. Salienta-se que o trabalho pode ser alargado a outros tipos de disposições regulamentares de forma a perceber quais são passíveis de verificação automática e identificar os requisitos de modelação aplicáveis.

\section{Agradecimentos}

Os autores agradecem o apoio pró-ativo da Gaiurb EM, quer nas várias discussões tidas sobre a temática que permitiu melhor compreender a realidade do licenciamento os caminhos a apontar, quer na disponibilização de informação relativamente ao caso de estudo analisado.

\section{Referências}

[1] J. P. P. Rodrigues, "Utilização De Modelos Bim Para Verificação Automática De Projetos,” Tese de Mestrado, Faculdade de Engenharia da Universidade do Porto, Porto, Portugal, 2015.

[2] Governo de Portugal, Regime Jurídico de Urbanização e de Edificação (RJUE) - Decretolei $n^{\circ}$ 555/99, de 16 de dezembro, alterado e republicado pelo Decreto-lei $n^{\circ}$ 136/2014, de 9 de setembro. Portugal: Diário da República, 2014.

[3] J. P. Poças Martins, "Modelação do Fluxo de Informação no Processo de Construção," Tese de Doutoramento, Faculdade de Engenharia da Universidade do Porto, Porto, Portugal, 2009.

[4] M. Anson, Y. H. Chiang, and J. Raftery, The Construction Sector in the Asian Economies, 1 edition. Abingdon, Oxon: Routledge, 2004.

[5] T. Krijnen and V. Berlo, "Methodologies for requirement checking on building models: a technology overview," in Design and Decision Support Systems in Architecture and Urban Planning - 13th International Conference on Design and Decision Support Systems in Architecture and Urban Planning, DDSS 2016, 2016, pp. 1-11.

[6] L. Manzione, "Ainda aprovamos projetos assim!," 4a, São Paulo, pp. 1-4, Nov-2015.

[7] R. Torreão, I. Mainardi, and T. Ricotta, "Code checking verificacao de regras na prefeitura de Salvador,” São Paulo, Brasil, 2019.

[8] Governo de Portugal, Regulamento Geral das Edificações Urbanas (RGEU) - Decreto-Lei 
n. 38 382, de 7 de Agosto de 1951, Alterado e republicado pelo Decreto-Lei $n .^{\circ}$ 220/2008, de 12 de Novembro. Portugal: Diário da República, 2008.

[9] C. Eastman, J. min Lee, Y. suk Jeong, and J. kook Lee, "Automatic rule-based checking of building designs," Autom. Constr., vol. 18, no. 8, pp. 1011-1033, 2009.

[10] A. I. de B. M. Neto, "Verificação de regras para aprovação de projetos de arquitetura em BIM para estações de metrô," Tese de Mestrado, Universidade de São Paulo, São Paulo, Brasil, 2016.

[11] N. A. Gualberto, "Implementação BIM ao nível do município, com foco no licenciamento: desafios e propostas.," Tese de Mestrado, Universidade do Minho, Guimarães, Portugal, 2019.

[12] Governo de Portugal, Regime Excecional para a Reabilitação Urbana (RERU) - DecretoLei $n^{\circ}$ 53/2014. Portugal: Diário da República, 2014, pp. 2337-2340. 